This document is the accepted manuscript version of the following article:

Niemelä, J. P., Rohbeck, N., Michler, J., \& Utke, I. (2020). Molecular layer deposited alucone thin films from long-chain organic precursors: from brittle to ductile mechanical characteristics. Dalton Transactions.

https://doi.org/10.1039/D0DT02210A

Received 00th January 20xx, Accepted 00th January 20xx

DOI: $10.1039 / \times 0 \times x 00000 x$

\title{
Molecular layer deposited alucone thin films from long-chain organic precursors: from brittle to ductile mechanical characteristics
}

\author{
Janne-Petteri Niemelä, ${ }^{* a}$ Nadia Rohbeck ${ }^{a}$, Johann Michler ${ }^{a}$ and Ivo Utkea
}

\begin{abstract}
Molecular layer deposition (MLD) is a strongly emerging thin-film technique for deposition of ultra-thin inorganic-organic hybrid ("metalcone") coatings directly from the gas phase, even on complex three-dimensional surfaces. In particular alucones (Al-based hybrids) have been found interesting e.g. for Li-ion battery and gas-barrier applications owing to the promise for enhanced mechanical performance provided by the organic fragments in the materials' structure. However, the metalcones based on short/small organic fragments are relatively brittle from the mechanical perspective. Here, we demonstrate an efficient approach for tailoring mechanical properties of MLD-fabricated hybrid inorganic-organic thin films through control over the organic precursor chain length. The proof-of-concept data is presented for alucones prepared using trimethyl aluminum together with 1,6-hexanediol or 1,10-decanediol as the precursors. Tensile testing coupled with in-situ optical microscopy reveals a gradual increase in stretchability with the increasing chain length, such that the crack onset strain value of $9.9 \pm 0.2 \%$ is obtained for the 1,10-decanediol-based 100 -nm-thick film. Through the demonstration of substantially suppressed crack propagation-as a sign of brittle-to-ductile transition-and the decrease in the elastic modulus value down to $4.6 \pm 2.1 \mathrm{GPa}$, the mechanical performance of the alucone family is extended to the polymeric regime. The substantial increase in the mechanical performance within the metalcone material family, makes the results particularly interesting for high-capacity high-volume-change battery electrodes requiring mechanically highly robust coatings.
\end{abstract}

\section{Introduction}

Molecular layer deposition (MLD) is a strongly emerging thinfilm technique for deposition of ultra-thin inorganic-organic hybrid and all-organic nanoscale coatings on a wide variety surfaces directly from the gas phase. ${ }^{1-4}$ The film deposition is carried out through cycles of self-limiting surface reactions, molecular monolayer-by-monolayer, such that even very highaspect-ratio nanostructures can be coated/infiltrated with subnanometer thickness control. The MLD routes have been established for various conventional (all-organic) polymers, such as polyimides and polyamides, and for hybrid materials ("metalcones"), whose structures often comprise M-O-R repeating units, where $M$ refers to a metal atom, $O$ is oxygen, and $\mathrm{R}$ is a short/small organic fragment. Typical metal sources are volatile precursors such as trimethyl aluminum, ${ }^{2}$ diethylzinc ${ }^{5}$ and $\mathrm{TiCl}_{4}{ }^{6,7}$ while the organic fragments are often deposited using organic alcohols ${ }^{2,5,7}$ or carboxylic acids ${ }^{3}$ as the precursors. As the MLD chemistries are based on ligand exchange reactions similar to those for atomic layer deposition

a. Mechanics of Materials and Nanostructures Laboratory, Empa (Swiss Federal Laboratories for Materials Science and Technology), Feuerwerkerstrasse 39, CH3602 Thun, Switzerland.

* janne-petteri.niemelae@empa.ch.

Electronic Supplementary Information (ESI) available: [Illustrative XRR data: Figure S1. Optical micrographs of the thin film surfaces under various strain states are provided: Figure S2 for the hexanediol-based material and Figure S3 for the decanediol-based material.]. See DOI: 10.1039/x0xx00000x
(ALD) of conformal all-inorganic materials, ${ }^{8}, 9$ these two techniques can be combined-in a single deposition chamberfor atomic/molecular level tailored hybrid multilayers, superlattices and alloys. ${ }^{10-12}$ Out of the metalcone family, alucones (Al-based hybrids) have received particular attention owing their promise for Li-ion-battery applications, ${ }^{13-15}$ and for flexible gas-barrier coatings as multilayers/alloys mixed with inorganic $\mathrm{Al}_{2} \mathrm{O}_{3}, 4,16,17$ For both of these applications mechanical robustness is a key material quality required. In particular, as an artificial solid-electrolyte interphase layer on Si-nanoparticle Liion-battery anodes, the alucone coatings would have to tolerate extreme volume changes of $300 \%{ }^{14}$

The research on the mechanical properties of the metalcones has followed the general trend in the field and focused on materials deposited from precursors with short straight-aliphatic (e.g. 1,2-ethanediol), ${ }^{2,18-20}$ branched-aliphatic (e.g. 1,2,3-propanetriol) and aromatic (e.g. 1,4-benzenediol)7, 21 carbon backbones. The mechanical properties of the metalcone thin films have been primarily characterized through nanoindentation ${ }^{18,} 20$ and tensile testing ${ }^{17}, 19$. Nanoindentation studies have shown that elastic modulus and hardness of the hybrid films can be decreased in comparison to the parent oxides, ${ }^{18,} 20$ while tensile experiments have indicated that stretchability and flexibility can be increased compared to oxides, $^{17,} 19$ and tailored in nanolaminate/superlattice structures. ${ }^{17,} 22$ The highest reported value for the crack onset strain (COS) for alucones is $1.8 \%$ for (300-nm) films deposited via the trimethyl aluminum/1,2-ethanediol process, and the 
fracture behavior is still characteristic for brittle materials. ${ }^{19}$ Even being relatively brittle, the short-chain alucones have been shown to improve the mechanical integrity of the $\mathrm{Si}$ nanoparticle electrodes and the capacity retention for Li-ion batteries. ${ }^{13,14}$ However, as for organic compounds the number of conformations (rotation about a single $\mathrm{C}-\mathrm{C}$ bonds) increases with increasing carbon chain length, increasing the carbon chain length of the organic MLD precursors provides us with an unexplored but a rational route for enhancing the mechanical properties of the metalcone thin films.

Here we demonstrate via tensile experiments that the COS value is enhanced up to $9.9 \%$ for $100-\mathrm{nm}$ thick alucone films using 1,10-decanediol as the long carbon-chain organic precursor. In-situ optical microscopy indicates that the crack propagation is suppressed with increasing chain length of the organic precursors and consequently a brittle-to-ductile transition is observed. Therefore, as is also reflected by the decrease of the elastic modulus values down to $4.6 \mathrm{GPa}$, the mechanical performance of the alucone material family is extended to the polymeric regime. Through geometrical extrapolation, the enhancement for the mechanical properties translates to tolerance of $300 \%$ volume expansions for batteryrelevant few-nm coating thickness.

\section{Experimental}

The alucone thin films were deposited via MLD in a laboratorybuilt ALD reactor at $130{ }^{\circ} \mathrm{C}$ on Si and on $50 \mu \mathrm{m}$-thick polyimide (kapton $200 \mathrm{HN}$ ) substrates. Trimethyl aluminum (TMA; Sigma Aldrich $97 \%$ ) was used as the Al metal precursor, 1,6hexanediol (HD) and 1,10-decanediol (DD; Sigma Aldrich $97 \%$ ) as the organic precursors. The organic precursors were heated to $120^{\circ} \mathrm{C}$ for sufficient volatility, while trimethyl aluminum was kept at room temperature. The MLD process parameters were studied for the TMA/HD and TMA/DD processes, as is described in the results and discussion section. Following the study, the dose/purge times for TMA, HD and DD were fixed as $0.1 \mathrm{~s} / 120$ $\mathrm{s}, 6 \mathrm{~s} / 120 \mathrm{~s}$ and $6 \mathrm{~s} / 120 \mathrm{~s}$, respectively. The TMA/HD and TMA/DD processes exhibit growth-per-cycle (GPC) values of 0.32 and $0.37 \mathrm{~nm}$, respectively, as determined from the slope of the fit to the thickness vs. cycle data. Around 100-nm films were deposited for the FTIR characterization (Si substrates) and for the tensile testing (polyimide substrates) of the materials, while $a \sim 400 \mathrm{~nm}$ film was deposited for the nanoindentation studies (Si substrate).

Thickness and density of the films were obtained by using the X-ray reflectivity (XRR, Bruker D8 Discover) technique for the films deposited on silicon substrates. The incident beam ( $\mathrm{Cu}$ $\mathrm{K} \alpha$ ) was conditioned by a Göbel mirror, a $0.1^{\circ}$ divergence slit and $0.1^{\circ}$ anti-scatter slit. The measurements were done in $\theta$ $2 \theta$ geometry over $2 \theta$ range of $0.1-5^{\circ}$ and the reflectivity patterns were analyzed by fitting the data to a physical model using DIFFRAC LEPTOS (Bruker) software. Chemical bonding in the films was studied via the Fourier transform infrared (FTIR; Perkin Elmer, Spectrum Two). The spectrometer was operated in the transmission mode subtracting the gas and the $\mathrm{Si}$ substrate backgrounds. A baseline correction was applied and the data was transformed from transmittance to absorbanche

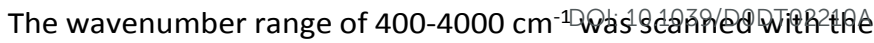
resolution of $4 \mathrm{~cm}^{-1}$ and the data was accumulated over 64 scans.

Uniaxial tensile testing of the films coated on the polyimide substrates was carried out using a tensile stage (MTI 8000-0010) equipped with a digital optical microscope (Keyence 500F) for in-situ monitoring of the fragmentation process. The tensile stage was operated at a constant strain rate of $1.4 \times 10^{-4} \mathrm{~s}^{-1}$ by means of displacement control. Microscope images were recorded at strain intervals of $1.4 \times 10^{-4}$ for the subsequent analysis. The strain values were determined via digital image correlation by tracking the distance between pairs of points (polyimide features) on the sample surface. The gauge sections of the samples were $5 \mathrm{~mm} \times 17 \mathrm{~mm}$. The regular imaging rate $\left(\mathrm{s}^{-1}\right)$ at strain intervals of $1.4 \times 10^{-4}$ enabled monitoring of the crack propagation speed.

To probe for the relevance of our tensile results for the $\mathrm{Li}$ ion battery field, the tolerance to the volume expansion on a Sinanoparticle electrode was estimated through a geometrical conversion and a thickness extrapolation of the measured crack onset strain (COS). The critical volume change was calculated via $\Delta V / V_{0}=\left(\Delta L / L_{0}+1\right)^{3}-1$, where $V_{0}$ is the initial volume of a spherical particle (prior to lithiation) and $\Delta V$ is the volume change (upon lithiation). $L_{o}$ is the length of a geodesic on surface of the sphere, while $\Delta L$ is the corresponding change; $\Delta L / L_{O}$ was assumed equivalent to uniaxial strain and to $\operatorname{COS}$ to obtain the critical volume change. The battery relevant COS value at few$\mathrm{nm}$ film thickness $(t)$ was obtained through an extrapolation from $100 \mathrm{~nm}\left(t_{0}\right)$ by using the known thickness dependence of $\cos : \cos (t)=\cos \left(t_{0}\right) \times\left(t_{0} / t\right)^{1 / 2,23}$ which has been shown to hold well at least down to $5-\mathrm{nm}$ film thickness for ALD $\mathrm{Al}_{2} \mathrm{O}_{3} \cdot{ }^{24}$

The elastic modulus and hardness were obtained using a Hysitron Ubi Nanoindenter equipped with a diamond Berkovich indenter tip, for a $400 \mathrm{~nm}$-thick DD-based alucone film on $\mathrm{Si}$ substrate. Various individual measurements were performed with maximum load ranging from 10 to $40 \mu \mathrm{N}$, such that the maximum penetration depth did not exceed $40 \mathrm{~nm}$. The uncertainty for the elastic modulus and hardness values represents the standard deviation over 50 individual measurements. The loading and unloading was done over $2 \mathrm{~s}$ and the maximum load was held for $1 \mathrm{~s}$ before unloading. The tip area function had been calibrated for low contact depths using fused silica beforehand. The elastic modulus and hardness values were determined by applying the Oliver and Pharr approach with 0.33 for the Poisson ratio of the alucone film.

\section{Results and discussion}

The MLD characteristics of the TMA/HD and TMA/DD processes were studied through ex-situ XRR thickness measurements for the films deposited on Si substrates (Figure 1; see Figure S1 for an example XRR pattern). The GPC values for both the processes show saturating behaviour with respect to the dose time of the organic precursors, such that the saturation of the surface reactions is observed around $2 \mathrm{~s}$ of the HD dose time, while a dose time of around $6 \mathrm{~s}$ is required for the DD precursor (Figure 

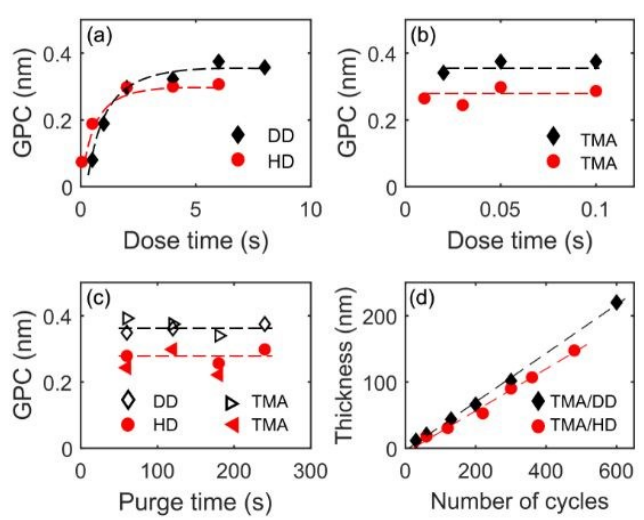

Figure 1. The MLD characteristics for thin films deposited from trimethylaluminium (TMA), 1,6-hexanediol (HD) and 1,10decanediol (DD) precursors. The growth-per-cycle (GPC) values as a function of the dose time: (a) for HD and DD, and (b) for TMA ("solid $\diamond$ " with DD, "solid o" with HD). (c) The purge-time dependence of the GPC values for all the precursors ("open $\diamond$ " and "open $\triangleright$ " the TMA/DD process, "solid o" and "solid $\triangleleft$ " the TMA/HD process). (d) Films' thickness vs. number of the MLD cycles. When not specifically studied, the dose/purge times for TMA, HD and DD were fixed as $0.05 \mathrm{~s} / 120 \mathrm{~s}, 6 \mathrm{~s} / 120 \mathrm{~s}$ and $6 \mathrm{~s} / 120 \mathrm{~s}$, respectively. The dashed lines serve as guides to the eye in (a-c) and represent linear fits to the data in (d). 1a). For TMA the surface reactions saturate below the studied dose times, owing to the high vapour pressure 1OFTIDPA TFRaUPe 1b). The GPC values are independent on the purge times indicating absence of substantial (parasitic) physisorption and gas-phase reactions in the studied purge-time range (Figure 1c). Thickness of the films increases linearly with increasing number of deposition cycles; the GPC values of 0.32 and $0.37 \mathrm{~nm}$ are obtained from the slopes of linear fits to the thickness vs. cycles data for the TMA/HD and TMA/DD processes, respectively. As the density functional theory calculations of Park et al. indicated height of $0.84 \mathrm{~nm}$ for the upright structural unit corresponding to one TMA/HD cycle, ${ }^{25}$ the organic fragments in our hybrid films are thus oriented in a rather tilted configuration on average and "double reactions" (both of the $-\mathrm{OH}$ groups of a diol molecule reacting with the surface $-\mathrm{CH}_{3}$ groups) can probably take place. Park et al. observed a decreasing slope for the thickness-vs-cycles curve after reaching a thickness of $60 \mathrm{~nm}$ for the TMA/HD process (slow-down/stop of the film growth), and ascribed this to the double reactions; that result hints that the MLD processes based on long-chain organic diols with flexible monomer backbones could be problematic. In our case the thickness increase is linear for the studied thickness range at least up to values as high as $200 \mathrm{~nm}$. Moreover, from the XRR data we extract the density values for our HD and DD-based films as $1.39 \pm 0.03$ and $1.31 \pm 0.05 \mathrm{~g} / \mathrm{cm}^{3}$. These values fit and extend the literature trend for alucones $\left(1.5-1.7 \mathrm{~g} / \mathrm{cm}^{3}\right.$ for $1,2-$ ethanediol, ${ }^{2,19,25} 1.42 \mathrm{~g} / \mathrm{cm}^{3}$ for 1,4-butanenediol, ${ }^{25}$ and 1.35 $\mathrm{g} / \mathrm{cm}^{3}$ for 1,6 -hexanenediol ${ }^{25}$ ), as expected for increasing share of lighter-than-Al elements $\mathrm{C}$ and $\mathrm{H}$. The density values for the long-chain alucones fall in the regime typical for polymeric materials (e.g. polyesters and epoxies). ${ }^{26}$ Our XRR data therefore indicates that hybrid inorganic-organic thin films can be grown through well-controlled MLD-type processes using long-chain diols as the organic precursors.

The expected gas-surface reactions (for both the halfreactions) for the present MLD processes constitute of ligandexchange reactions between the $-\mathrm{CH}_{3}$ groups from TMA and $\mathrm{OH}$ groups from the organic diols, followed by release of $\mathrm{CH}_{4}$ as the by-product. Therefore, it is expected that the organic

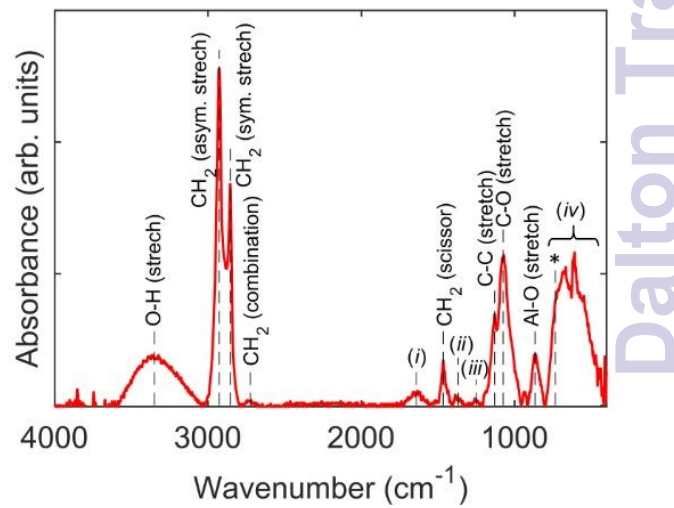

Figure 2. The Fourier transform infrared spectrum for the hybrid inorganic-organic film deposited through the TMA/DD MLD route. 
monomer units are bound together via O-Al-O linkages, and assuming each $\mathrm{Al}$ atoms to form three bonds, the material consists of repeating units of Al-[O- $\left.\left(\mathrm{CH}_{2}\right)_{x / 2}\right]_{3}$, where $x(\geq 2)$ is the number of carbon atoms in the organic precursor. The chemical bonding was characterized via FTIR spectroscopy in the wave number range of $400-4000 \mathrm{~cm}^{-1}$ for the DD-based hybrid material. It is can be seen that all the fingerprint spectral features for alucones based on aliphatic saturated hydrocarbon linkers are present (Figure 2). ${ }^{2}$ For the backbone of the material stretching modes of $\mathrm{Al}-\mathrm{O}, \mathrm{C}-\mathrm{O}$ and $\mathrm{C}-\mathrm{C}$ bonds are seen at 867, 1075 and $1133 \mathrm{~cm}^{-1}$, respectively. For the side groups, strong absorption maxima are observed at around 2855 and $2928 \mathrm{~cm}$ 1 for the symmetric and asymmetric $\mathrm{CH}_{2}$ stretching modes, together with weak $\mathrm{CH}_{2}$ peaks at 2725 and 1466 for the combinatorial stretching mode and the scissor mode, respectively. A broad maximum centered at $3400 \mathrm{~cm}^{-1}$ corresponds to the stretching mode of bonded $-\mathrm{OH}$, where the broadness is possibly contributed by hydrogen bonding. ${ }^{27}$ Probably the $-\mathrm{OH}$ groups represent a fraction of unreacted $-\mathrm{OH}$ groups that remain unavailable to react with TMA owing to steric hindrance posed by the organic precursor molecules. The absorption peak (i) at $1641 \mathrm{~cm}^{-1}$ can be partly ascribed to $\mathrm{C}=\mathrm{C}$ stretching mode of enolate-type vinyl ether species, ${ }^{2,} 27$ that could form through dehydration of the unreacted $-\mathrm{OH}$ groups, and it can also be partly contributed by a scissor mode of adsorbed $\mathrm{H}_{2} \mathrm{O} .{ }^{2}$ Peaks (ii) and (iii) at 1370 and $1250 \mathrm{~cm}^{-1}$ can be ascribed to $\mathrm{CH}_{2}$ twist and wag modes, where the former can also be contributed by a $\mathrm{COH}$ bending mode. ${ }^{2}$ The broad band (iv) between ca. 400 and $800 \mathrm{~cm}^{-1}$ is ascribed to phonon-like modes with O-AI-O character also found for TMA/EG-based alucones ${ }^{28}$

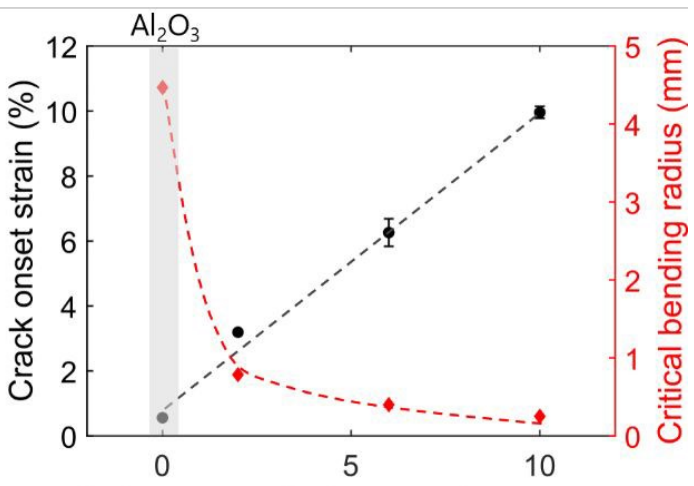

Carbon atoms in the organic monomer

Figure 3. The crack onset strain and the critical bending radius for the hybrid inorganic-organic films deposited on 50 - $\mu$ m-thick polyimide substrates through the TMA/EG, TMA/HD and TMA/DD MLD routes as a function of the number of the carbon atoms in the organic precursor. The data for all-inorganic $\mathrm{Al}_{2} \mathrm{O}_{3}$ is show for reference (at $C$ atom number $=0$ ). The crack onset strain error bars for the HD and the DD based materials represent the standard deviation; for the critical bending radius the standard deviations are within the marker size. All values are scaled to $100 \mathrm{~nm}$ film thickness ( $\cos \propto t_{f}^{-1 / 2}$ ). The data for the EG based material and $\mathrm{Al}_{2} \mathrm{O}_{3}$ were taken from our previous works. ${ }^{12,19}$ The dashed lines serve as guides to the eye. and for amorphous $\mathrm{AlO}_{x}{ }^{29}$ and to alcohol-related $\mathrm{gut}$ - of bend of $\mathrm{OH}$ at $720-590 \mathrm{~cm}^{-1} ; 27$ in particulapthe specterar Tweight at $720-750 \mathrm{~cm}^{-1}$ indicated with $\left(^{*}\right)$ could be ascribed to methylene $-\left(\mathrm{CH}_{2}\right)_{n}$ - rocking mode which only becomes active for $n \geq 3,{ }^{27}$ and would hence be indicative of our long carbon chain structure. For our DD-based hybrid the intensity of the $\mathrm{CH}_{2}$ stretching modes are higher relative to the intensity of the and $\mathrm{C}-\mathrm{O}$ and $\mathrm{Al}-\mathrm{O}$ peaks, in comparison to the EG-based films. ${ }^{2}$, 28,30 This naturally follows from the expected increase in carbon content for the DD-based film. The intensity of the $C-C$ stretching mode remains low as the dipole moments of adjacent $\mathrm{C}-\mathrm{C}$ bonds of the long aliphatic carbon chain may largely cancel out and hence contribute little to the net dipole moment and the related IR absorption.

Mechanical stretchability and flexibility of the films were studied via tensile testing coupled with in-situ optical microscopy for the films deposited on the polyimide substrates. The samples were strained at a constant strain rate without any visual changes in the film morphology until the observation of the first $\operatorname{crack}(\mathrm{s})$ at the crack onset strain (COS). Crack onset strain provides us with a direct metric of film stretchability, while flexibility of the film-substrate system can be described in terms of critical bending radius $R_{c}=\left(t_{s}+t_{f}\right) /(2 \mathrm{COS})$, where $t_{f}$ and $t_{s}$ are the thicknesses of the film and the substrate, respectively. Figure 3 shows the COS (for 100-nm film thickness) and the corresponding $R_{c}$ values (for $50-\mu \mathrm{m}$ substrate thickness) as a function of the carbon atoms in the organic precursor for our MLD-fabricated alucone films; the reference data for ALDfabricated $\mathrm{Al}_{2} \mathrm{O}_{3}$, and alucone fabricated trough the TMA/1,2ethanediol MLD process are from our previous works. ${ }^{19,} 31$ The COS values show remarkable progressive increase with the chain length of the organic precursor, such that the value of $9.9 \pm 0.2 \%$ is achieved for the film grown using the DD precursor. This value is over an order-of-magnitude higher in comparison to that for the all-inorganic $\mathrm{Al}_{2} \mathrm{O}_{3}$ thin film, and is $310 \%$ (relative) higher than the state-of-the-art for the MLDfabricated hybrid materials (the data for the 1,2-ethanediolbased material). The COS value of $9.9 \%$ corresponds to $0.252 \pm 0.005-\mathrm{mm} R_{c}$ on the $50-\mu \mathrm{m}$ substrate, relevant for rollto-roll processing and flexible/foldable barrier applications, and-most interestingly-it translates to the desired critical volume expansion of $300 \%$ for battery-relevant 3-nm coating thickness on a nanoparticle surface. The data represents a proof-of-concept for tailoring mechanical stretchability and flexibility of MLD-fabricated hybrid inorganic-organic materials through the control over the organic precursor chain length.

The fragmentation process of brittle materials is typically characterized by formation of channel cracks perpendicular to the (uniaxial) straining axis. ${ }^{23,32}$ After the crack onset strain the crack density along the straining axis first increases exponentially with increasing strain and then eventually saturates. ${ }^{23}, 32$ In this process (a tip of) an individual crack propagates rapidly across long distances, even across the whole width of the film. We observe this type of brittle fracture for $\mathrm{Al}_{2} \mathrm{O}_{3}$ and for the EG-based hybrid films, and in our recent works we deduced through Weibull statistics e.g. that the EG-based hybrid shows cohesive strain and interfacial shear 


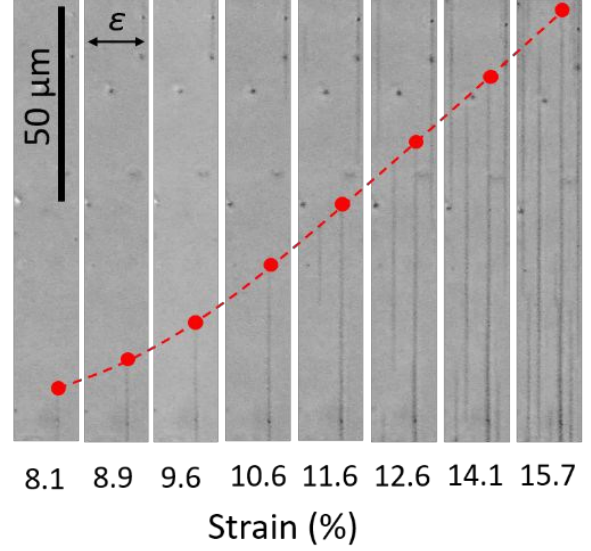

Figure 4. Propagation of a crack tip with increasing tensile strain $(\varepsilon)$ as followed through the in-situ optical microscopy and indicated by the dashed line and the round markers. The micrograph series is for the hybrid inorganic-organic film deposited through the TMA/HD route and having 6 carbon atom chains in the linker unit.

strain/strength values superior to those for $\mathrm{Al}_{2} \mathrm{O}_{3}$, while $\mathrm{Al}_{2} \mathrm{O}_{3}$ has higher cohesive strength owing to its higher elastic modulus. ${ }^{19,} 31$ Remarkably, the mechanical behavior of our hybrid materials based on the long HD and DD precursors progressively departs from the picture of brittle fracture. After the crack onset strain more cracks are formed with increasing strain, however, the propagation of the crack tips becomes considerably slow, such that it can be captured with the optical microscope (Figures 4, S2 and S3). The crack propagation rate of $9.4 \pm 0.6 \mu \mathrm{m} / \mathrm{strain} \%$ (or $0.13 \pm 0.01 \mu \mathrm{m} / \mathrm{s}$ ) is obtained for the HD-based films, with the crack length eventually exceeding the field of view of $200 \mu \mathrm{m}$ (Figure 5). For the DD-based material the crack propagation initiates with a rate similar to that for the HD-based materials, but after a short period of propagation over a strain interval of around $2 \%$ the propagation stops at a tip-to-tip crack length of $\sim 10 \mu \mathrm{m}$. The slowing down of the crack propagation probably links to the elastic strain energy being increasingly dissipated through plastic deformation of the polymer chains, which is typical for ductile polymers. ${ }^{33,} 34$ It is expected that such deformation processes become increasingly available for our long-chain hybrid materials through a higher number of possible conformations (rotation about $\mathrm{C}-\mathrm{C}$ single bonds), ${ }^{35}$ when comparing to MLD films deposited from short chain precursors. As the elastic mismatch between the film and the substrate decreases for the films with long-chain molecules (elastic modulus for the DD-based film of $4.6 \mathrm{GPa}$ approaches $2.5 \mathrm{GPa}$ for kapton $200 \mathrm{HN}$; see also Figure 6), plastic deformation of the substrate may also increasingly contribute to the dissipation of the energy released in the cracking process and slow down the crack propagation. ${ }^{36}$
For uniaxially strained ductile polymer materials ${ }_{A} a_{i c}$ typical

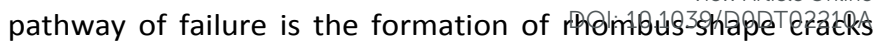
("microvoids") with a characteristic width $(w)$ and length (l), I being orthogonal to the straining axis. ${ }^{33,34,37}$ Recently, Alkhadra et al. studied the strain evolution of such microvoids for a series of semiconducting polymers in terms of $(I / w) / \varepsilon$, a quantity that they coined as microvoid propagation number $(m)$, and which by describing material's resistance to fracture propagation is an effective measure of ductility. ${ }^{37}$ In our case the majority of the shape evolution of the cracks is in the direction orthogonal to the straining axis (I). From the optical micrographs the crack width $(w)$ is estimated to be $\sim 1-2 \mu \mathrm{m}$ with moderate evolution with increasing strain (note that $\sim \mu \mathrm{m}$ approaches the resolution limit of our optical microscope, and thus the crack width is not resolved with high precision). A rough estimation for the $m$ value of our HD-based hybrid material is $\log _{10}(m) \sim 3$. This is yet higher than $\log _{10}(m) \lesssim 2$ observed for a series of ductile semiconducting polymers, and implies that cohesion and ductility are still lower for our HD-based hybrid material in comparison to ductile polymers. ${ }^{34,} 37,38$ In comparison to the HD-based material, our DD-based hybrid shows more ductile behavior as the propagation of the cracks essentially stops soon after their formation (while the cracks are still isolated). For the DD-based material the crack-to-crack distance, however, becomes small above $\sim 12 \%$ strain, such that the cracks cannot anymore be considered isolated, and interaction between the cracks can influence the crack propagation. Interestingly, our hybrid inorganic-organic thin films show COS vs. crack propagation behavior similar to what has been observed for polymer thin films: brittle fracture with rapid crack propagation for $\operatorname{COS}<5 \%$ and lower tendency for crack propagation, and hence qualitatively greater fracture toughness, for $\operatorname{COS}>5 \%{ }^{34}$ Increase in fracture toughness is desired for alucone $/ \mathrm{Al}_{2} \mathrm{O}_{3}$ multilayer architectures, ${ }^{24}$ relevant e.g. for moisture barrier applications. From the general applications perspective, the slowing down of the crack propagation seen for our HD and DD-

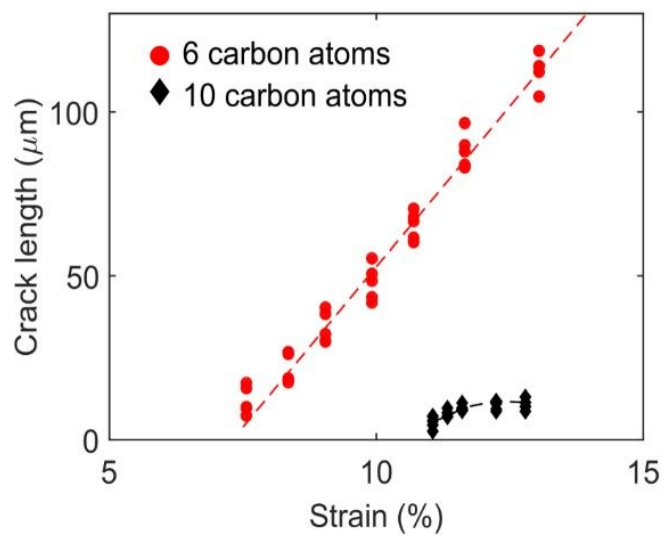

Figure 5. The crack length as a function of strain for the hybrid inorganic-organic films deposited through TMA/HD and TMA/DD MLD routes, with 6 and 10 carbon atom chains in their linker units, respectively. Evolution of five cracks was followed for both the samples. The dashed lines serve as guides to the eye. 
based materials will be beneficial as the mechanical failure for these materials will not be as catastrophic as it is for brittle inorganic materials or hybrid thin films based on short-chain MLD precursors.

Nanoindentation studies were carried out for our DD-based material in order to further study the mechanical properties of hybrid MLD materials based on long-chain organic precursors. A series of load-controlled indents were performed, such that the maximum penetration depth of the indenter tip was between 20 and $40 \mathrm{~nm}$; Figure 6 shows a representative load vs. displacement graph from these experiments. Upon indentation the film exhibits plasticity immediately, as observed also for alucone films based short-chain precursors. ${ }^{19}$ At the same time the constant load hold at maximum (over one second) produces a significant creep response illustrating the highly viscoelastic behavior of the material. The elastic modulus and hardness

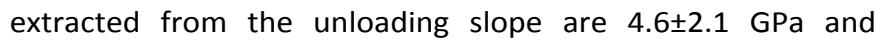
$0.44 \pm 0.12 \mathrm{GPa}$. The elastic modulus is nearly an order-ofmagnitude lower than the values in the range of 21-44 GPa that have been previously reported for short-chain MLD alucones or metalcones in general. ${ }^{19-21}$ The hardness is also at the lower end of the previously obtained values around $1 \mathrm{GPa}$ for the MLD metalcones. ${ }^{19-21}$ While the elastic modulus values for the shortchain metalcones of few tens of GPa are still characteristic for composite materials ( 15-200 GPa), our approach of using longchain organic precursor extends the properties of the metalcone material family to the polymeric regime of elasticity ( 0.1-10 GPa). ${ }^{26}$ Overall, the nanoindentation experiment corroborates our observations from the tensile experiments, that is, the alucone materials deposited using long-chain organic precursors exhibit substantially enhanced mechanical properties (for e.g. battery applications) in comparison to the alucones deposited using short organic precursors (or inorganic materials).

\section{Conclusions}

We have presented an efficient approach for tailoring mechanical properties of molecular layer deposited hybrid inorganic-organic thin films through control over the organic precursor chain length. The proof-of-concept data has been presented for Al-based hybrid materials (alucones), prepared using trimethyl aluminum together with 1,6-hexanediol or 1,10decanediol as the precursors. Through this approach we have demonstrated a factor-of-three (or larger) enhancement in stretchability, flexibility and elasticity for nanoscale alucone thin films - a material family that has recently been found of high technological interest e.g. for Li-ion-battery and gas-barrier applications, where the robust mechanical performance of the thin-film coatings is a key. For example, the obtained $9.9 \%$ crack onset strain for 100-nm films extrapolates to tolerance of 300 $\%$ volume expansion at a film thickness of $3 \mathrm{~nm}$, relevant for the Li-ion battery applications. We believe that the approach can be generalized for other molecular layer deposited thin films based on metals other than Al, i.e. for a wide variety of metalcone materials, that can also be prepared from long-chain organic precursors other than alcohols, such as carboxylic acids and amines. Therefore the approach is foreseen to be relexant for

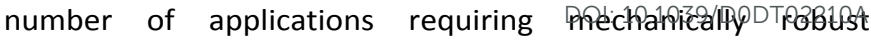
conformal nanoscale thin-film coatings.

\section{Conflicts of interest}

There are no conflicts to declare.

\section{Acknowledgements}

We acknowledge funding by the Empa internal project IRC1829. EMPAPOSTDOCS-II programme is also acknowledged; it has received funding from the European Union's Horizon 2020 research and innovation programme under the Marie Skłodowska-Curie grant agreement (754364).

\section{References}

1 B. H. Lee, M. K. Ryu, S. Choi, K. Lee, S. Im and M. M. Sung, J. Am. Chem. Soc., 2007, 129, 16034.

2 A. A. Dameron, D. Seghete, B. B. Burton, S. D. Davidson, A. S. Cavanagh, J. A. Bertrand and S. M. George, Chem. Mater. 2008, 20, 3315.

3 O. Nilsen, K. B. Klepper, H. Ø Nielsen and H. Fjellvåg, ECS Trans., 2008, 16 (4), 3.

4 X. Meng, J. Mater. Chem. A, 2017, 5, 18326

5 M. Aghaee, J.-P. Niemelä, W. M. M. Kessels and M. Creatore, Dalton Trans., 2019, 48, 3496.

6 A. I. Abdulagatov, R. A. Hall, J. L. Sutherland, B. H. Lee, A. S. Cavanagh and S. M. George, Chem. Mater., 2012, 24, 2854.

7 J.-P. Niemelä and M. Karppinen, Dalton Trans., 2015, 44, 591.

8 L. Ma, R. B. Nuwayhid, T. Wu, Y. Lei, K. Amine and J. Lu, Adv. Mater. Interfaces, 2016, 3, 1600564.

9 M. Knez, K. Nielsch and L. Niinistö, Adv. Mater., 2007, 19, 3425.

10 J.-P. Niemelä, A. J. Karttunen and M. Karppinen, J. Mater. Chem. C, 2015, 3, 10349.

11 J.-P. Niemelä, A. Giri, P. E. Hopkins and M. Karppinen, J. Mater. Chem. A, 2015, 3, 11527.

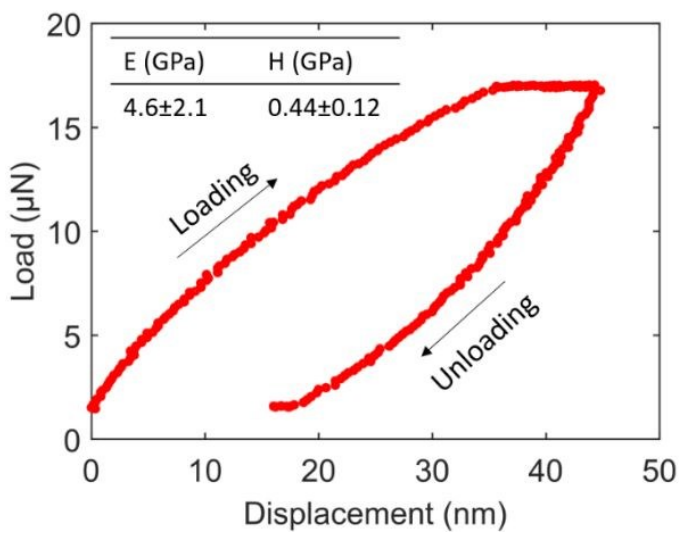

Figure 6. A characteristic load-displacement nanoindentation graph for the DD-based hybrid material. The elastic modulus $(E)$ and the hardness $(H)$ values averaged over various indents are also shown. 
12 J.-P. Niemelä, M. Aghaee, W. M. M. Kessels, M. Creatore and M. A. Verheijen, J. Vac. Sci. Technol. A, 2019, 37, 040602.

13 D. Molina Piper, Y. Lee, S. Son, T. Evans, F. Lin, D. Nordlund, X. Xiao, S. M. George, S. Lee and C. Ban, Nano Energy, 2016, 22, 202.

14 D. M. Piper, J. J. Travis, M. Young, S. Son, S. C. Kim, K. H. Oh, S. M. George, C. Ban and S. Lee, Adv. Mater., 2014, 26, 1596.

15 Y. Zhao and X. Sun, ACS Energy Lett., 2018, 3, 899.

16 D. Choi, M. Yoo, H. M. Lee, J. Park, H. Y. Kim and J. Park, ACS Appl. Mater. Interfaces, 2016, 8, 12263.

17 S. Jen, B. H. Lee, S. M. George, R. S. McLean and P. F. Carcia, Appl. Phys. Lett., 2012, 101, 234103.

18 D. C. Miller, R. R. Foster, S. Jen, J. A. Bertrand, D. Seghete, B. Yoon, Y. Lee, S. M. George and M. L. Dunn, Acta Mater., 2009, 57, 5083.

19 M. Ruoho, N. Tarasiuk, N. Rohbeck, C. Kapusta, J. Michler and I. Utke, Mater. Today Chem., 2018, 10, 187.

20 B. H. Lee, B. Yoon, V. R. Anderson and S. M. George, J. Phys. Chem. C, 2012, 116, 3250.

21 B. Yoon, B. H. Lee and S. M. George, J. Phys. Chem. C, 2012, 116, 24784.

22 A. Philip, J.-P. Niemelä, G. Tewari, B. Putz, T. Edwards, M. Itoh, I. Utke and M. Karppinen, ACS Appl. Mater. Interfaces, 2020, 12(19), 21912.

23 Y. Leterrier, J. Andersons, Y. Pitton and J. A. E. Månson, J. Polym. Sci. B Polym. Phys., 1997, 35, 1463.

24 D. C. Miller, R. R. Foster, Y. Zhang, S. Jen, J. A. Bertrand, Z. Lu, D. Seghete, J. L. O'Patchen, R. Yang, Y. Lee, S. M. George and M. L. Dunn, J. Appl. Phys., 2009, 105, 093527.

25 Y. Park, H. Kim, B. Cho, C. Lee, S. Choi, M. M. Sung and J. S. Lee, ACS Appl. Mater. Interfaces, 2016, 8, 17489.

26 M. F. Ashby, Materials Selection in Mechanical Design, Elsevier Ltd., 2011.

$27 \mathrm{~J}$. Coates, Interpretation of Infrared Spectra, A Practical Approach in Encyclopedia of Analytical Chemistry, John Wiley \& Sons Ltd., 2006.

28 A. Perrotta, P. Poodt, F. J. van den Bruele, W. M. M. Kessels and M. Creatore, Dalton Trans., 2018, 47, 7649.

29 A. C. Dillon, A. W. Ott, J. D. Way and S. M. George, Surf. Sci., 1995, 322, 230.

30 K. Van de Kerckhove, M. K. S. Barr, L. Santinacci, P. M. Vereecken, J. Dendooven and C. Detavernier, Dalton Trans., 2018, 47, 5860.

31 M. Ruoho, J.-P. Niemelä, C. Guerra-Nunez, N. Tarasiuk, G. Robertson, A. A. Taylor, X. Maeder, C. Kapusta, J. Michler and I. Utke, Nanomaterials, 2020, 10(3), 558.

32 Y. Leterrier, L. Boogh, J. Andersons and J. A. E. Månson, J. Polym. Sci. B Polym. Phys., 1997, 35, 1449.

33 N. Balar and B. T. O'Connor, Macromolecules, 2017, 50, 8611.

34 B. Roth, S. Savagatrup, N. V. de los Santos, O. Hagemann, J. E. Carlé, M. Helgesen, F. Livi, E. Bundgaard, R. R. Søndergaard, F. C. Krebs and D. J. Lipomi, Chem. Mater., 2016, 28, 2363.

35 S. Fakirov, Fundamentals of Polymer Science for Engineers, Wiley-VCH, Weinheim, Germany, 2017.

36 J. L. Beuth and N. W. Klingbeil, J. Mech. Phys. Solids, 1996, 44, 1411.

37 M. A. Alkhadra, S. E. Root, K. M. Hilby, D. Rodriquez, F. Sugiyama and D. J. Lipomi, Chem. Mater., 2017, 29, 10139.

38 O. Awartani, B. I. Lemanski, H. W. Ro, L. J. Richter, D. M. DeLongchamp and B. T. O'Connor, Adv. Energy Mater., 2013, 3, 399. 
Table of contents

We tailor mechanical properties of molecular-layer-deposited inorganic-organic films through control over the organic precursor length, relevant for battery applications.

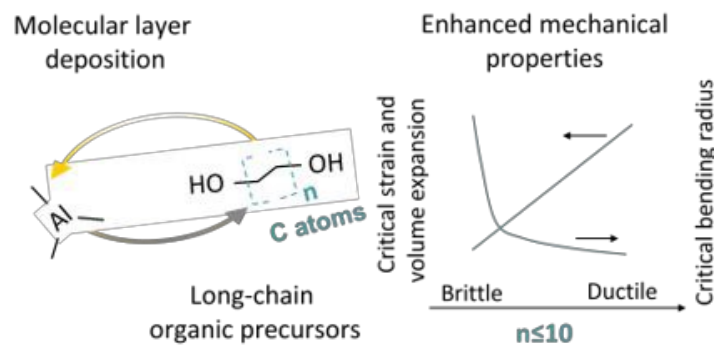




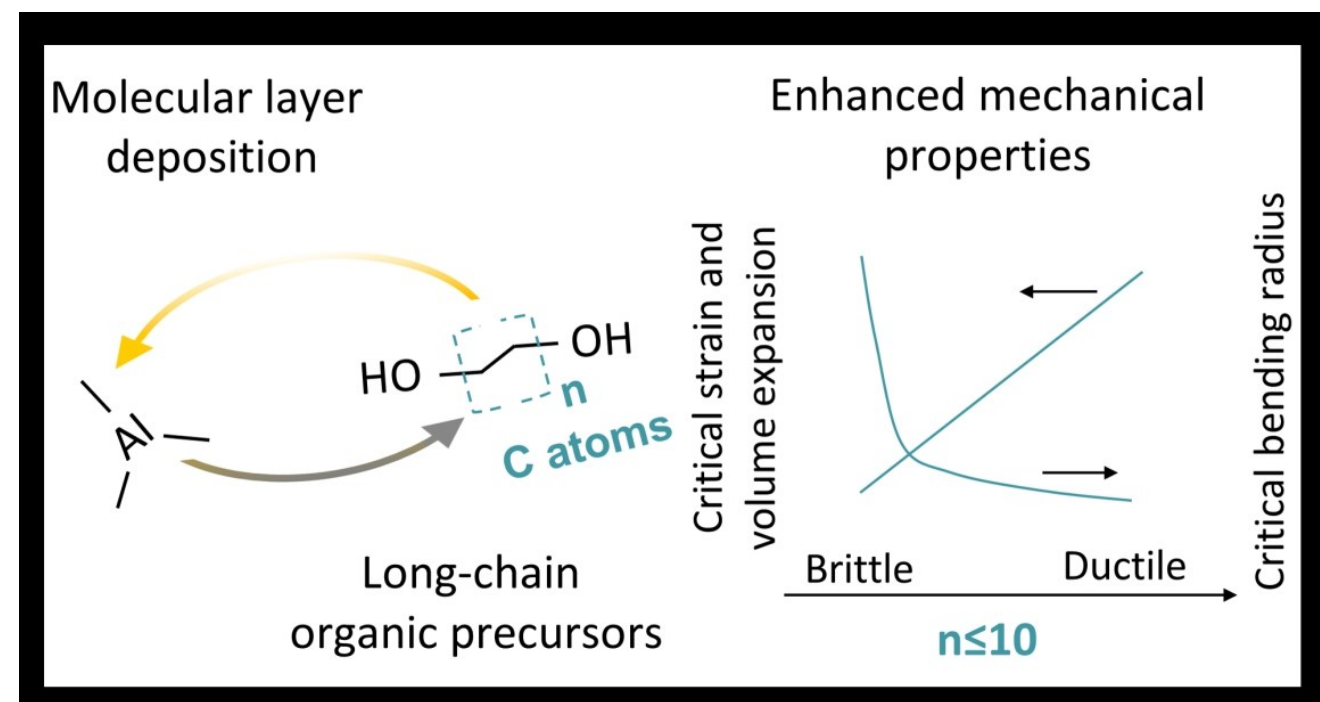

